\title{
UM PROCEDIMENTO SIMPLES E BARATO PARA A CONSTRUÇÃO DE UM EQUIPAMENTO "DIP-COATING" PARA DEPOSIÇÃO DE FILMES EM LABORATÓRIO
}

\author{
Alfredo R. M. Oliveira e Aldo J. G. Zarbin* \\ Departamento de Química, Universidade Federal do Paraná, CP 19081, 81531-990 Curitiba - PR
}

Recebido em 3/12/03; aceito em 9/7/04; publicado na web em 12/11/04

\begin{abstract}
A CHEAP AND SIMPLE PROCEDURE FOR BUILDING A DIP-COATING EQUIPMENT FOR THIN FILM DEPOSITS IN THE LABORATORY. In this work we show how to build a piece of equipment for depositing thin films by the dip-coating process, using inexpensive components easily found on the market. This equipment allows us to control the dipping and pulling velocity at which the substrate is put in the film precursor solution, two very important parameters for obtain thin films. This article discusses the construction of the mechanical and electric parts of the equipment, a simple method to interface it to a computer and the development of the software to control the dip-coating parameters.
\end{abstract}

Keywords: dip-coating; thin films; precursors.

\section{INTRODUÇÃO}

A obtenção de materiais na forma de filmes finos corresponde a um desafio científico e tecnologicamente importante, uma vez que filmes sólidos apresentam uma infinidade de aplicações nos mais diferenciados dispositivos ${ }^{1}$. Filmes podem ser depositados através de uma grande variedade de técnicas, que podem ser classificadas de acordo com a fase do meio contendo seu precursor. Na Figura 1 estão relacionadas algumas técnicas de deposição de filmes a partir de precursores em fase sólida, líquida e gasosa ${ }^{2}$. Maiores detalhes sobre as técnicas citadas na Figura 1 podem ser obtidos nas referências 2-8. As técnicas envolvendo deposição em fase líquida geralmente envolvem a dissolução de um precursor em um solvente adequado, seguida de sua deposição na superfície do substrato e subseqüente evaporação controlada do solvente e/ou tratamento térmico.

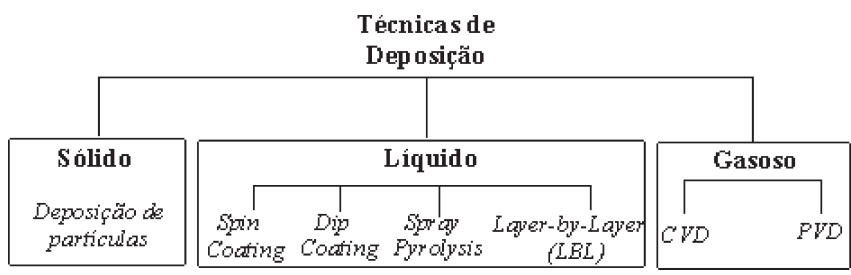

Figura 1. Diagrama dos principais métodos de obtenção de filmes a partir de precursores sólidos, líquidos e gasosos

A utilização de precursores em fase líquida apresenta um grande número de vantagens sobre outras rotas de deposição: i) grande variedade de precursores disponíveis; ii) estruturas complexas (ou partículas) podem ser obtidas em solução e depositadas sobre o substrato e iii) os equipamentos necessários para a deposição são mais simples e baratos que os análogos para a deposição a partir de precursores gasosos (como em um processo de CVD, por exemplo) ${ }^{2}$.

Uma das técnicas mais difundidas e utilizadas para o crescimento de filmes finos a partir de precursores em fase líquida é a técnica de "dip-coating", cujo princípio de funcionamento consiste em se mergulhar perpendicularmente o substrato dentro da solução contendo o

*e-mail: aldo@quimica.ufpr.br precursor e depois retirá-lo da mesma. O processo de inserção e retirada do substrato na solução deve ser realizado com velocidade controlada e constante e sem nenhum tipo de vibração ou interferência externa, de modo a garantir a deposição de um filme homogêneo. O tempo de permanência do substrato na solução anteriormente à sua retirada também é um fator de controle importante. Isto significa que para se obter filmes de qualidade, além das características do substrato e da solução precursora (solvente, concentração, viscosidade, tipo de precursor, etc.), é necessária a utilização de um equipamento que promova a inserção e a retirada do substrato com alta estabilidade, com controle fino da velocidade e livre de vibrações.

Neste trabalho apresentamos um método de construção de um equipamento para a deposição de filmes através da técnica de "dipcoating", utilizando materiais baratos e disponíveis no mercado nacional, alguns deles inclusive podendo ser re-aproveitados a partir de sucatas. Apresentamos, também, um método de interfaceamento deste equipamento a um computador do tipo PC e o desenvolvimento de um software para controle do mesmo.

\section{As definições do projeto}

De acordo com o mencionado anteriormente, um dos requisitos para que o filme tenha qualidade é que o movimento de inserção e retirada do substrato na solução seja suave e desprovido de vibrações. Também são necessários o controle das velocidades de imersão, de retirada e o tempo em que substrato permanece imerso na solução. O uso de um computador controlando um motor de passo é a maneira mais simples de atender a todos estes requisitos. Desta forma, a parte mecânica deste projeto é composta por um motor de passo, por um parafuso com rosca e por uma guia linear. A idéia é transformar o movimento rotacional do motor em um movimento linear de descida e subida, conforme mostra a Figura 2. Isto é feito fixando o parafuso com rosca na guia linear. Impedindo a sua rotação, a rosca irá se movimentar para cima ou para baixo, dependendo do sentido de rotação do parafuso.

A resolução do equipamento irá depender do número de fusos por centímetro que o parafuso possui. Aqui foi utilizado um parafuso que é muito comum e que possui 13 fusos por $\mathrm{cm}$. Como o motor de passo executa 200 passos para dar uma volta completa, a resolução será: $1 \mathrm{~cm} / 13 / 200=0,0038$ milímetros, ou seja, 3,8 $\mu \mathrm{m}$. 


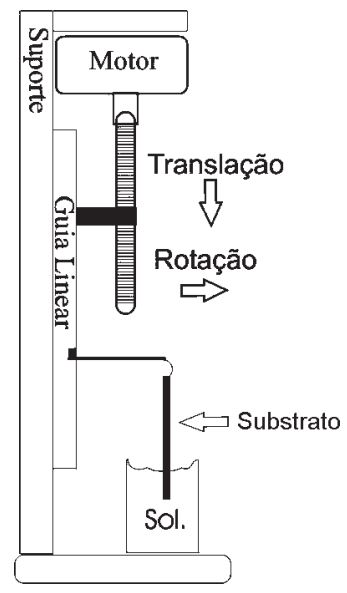

Figura 2. Diagrama utilizado na montagem do equipamento visando a conversão do movimento de rotação em translação

O motor de passo é um tipo de motor especial, construído de maneira que seu eixo avança apenas uma fração da circunferência a cada pulso. O motor utilizado neste trabalho foi retirado de um antigo "floppy disk drive" de 5" 1/4, possui duas bobinas (como mostrado na Figura 3) e são necessários 200 pulsos (ou passos) para dar uma volta completa no eixo. Os sinais são enviados às bobinas sempre em relação ao terra $(\mathrm{G})$ de cada bobina. Se assumirmos que cada ponto (A, A', B, B') pode estar ligado ou desligado, podemos criar a tabela lógica ilustrada na Tabela 1 , onde "1" significa ligado e " 0 ", desligado. Se a sequiência 1-4 faz com que o motor gire 4 passos para a direita, invertendo a seqüência de pulso fará o motor girar para a esquerda. Isto é feito pelo software e controla a direção em que o motor se move.

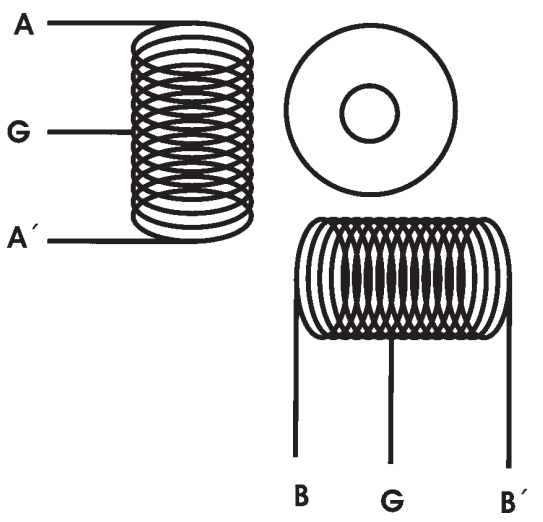

Figura 3. Diagrama da conexão de um motor de passo

Tabela 1. Seqüência de pulsos em motor de passo

\begin{tabular}{ccccc}
\hline Pulso & A & A $^{\prime}$ & B & B' $^{\prime}$ \\
\hline 1 & 1 & 0 & 0 & 0 \\
2 & 0 & 1 & 0 & 0 \\
3 & 0 & 0 & 1 & 0 \\
4 & 0 & 0 & 0 & 1 \\
\hline
\end{tabular}

\section{Eletrônica e interfaceamento}

Os computadores pessoais (PC) possuem dois tipos de interface de comunicação: a porta paralela e a porta serial, estando a diferença entre elas na maneira com que os dados são enviados. Neste artigo utilizaremos a porta paralela do computador para controlar o motor de passo utilizado no equipamento "dip-coating”. Para tal, segue uma rápida explicação do funcionamento da mesma.

O conceito básico para estabelecer a comunicação entre o computador e um equipamento externo (uma impressora, por exemplo) é o "byte". Um "byte" é composto por no mínimo oito "bits". Cada "bit" possui dois estados: ligado ou desligado, permitindo $256\left(2^{8}\right)$ combinações de estados discretos possíveis. A porta paralela normalmente é usada para conectar a impressora e no conector na parte de trás do computador existem 25 pinos que são distribuídos de acordo com o apresentado na Tabela 2. Podemos observar que os 25 pinos distribuídos em 4 funções distintas: 8 pinos de dados, 4 de controle, 5 de estado e 8 de aterramento. Os 8 pinos de dados são usados como saídas, os 5 pinos de estado podem ser usados como entrada de dados e os 4 pinos de controle também podem ser usados como saída. Na internet existem vários "sites" que tratam de maneira profunda o assunto, o que escapa dos objetivos deste artigo.

Tabela 2. Função dos pinos em uma porta paralela comum

\begin{tabular}{cll}
\hline $\begin{array}{c}\text { Número } \\
\text { do pino }\end{array}$ & Função & Tipo \\
\hline 1 & Sincronismo (STROBE) & Controle (CONTROL) \\
2 & Bit de dados 0 & Saída \\
3 & Bit de dados 1 & Saída \\
4 & Bit de dados 2 & Saída \\
5 & Bit de dados 3 & Saída \\
6 & Bit de dados 4 & Saída \\
7 & Bit de dados 5 & Saída \\
8 & Bit de dados 6 & Saída \\
9 & Bit de dados 7 & Saída \\
10 & Reconhecimento & Estado(STATUS) \\
& (AKNOWLEDGE) & \\
11 & Ocupado (BUSY) & Estado \\
12 & Sem papel (PAPER EMPTY) & Estado(STATUS) \\
13 & Impressora On Line & Estado(STATUS) \\
14 & Avanço de linha (CR) & Controle (CONTROL) \\
15 & Erro (ERROR) & Estado(STATUS) \\
16 & Iniciar (INITIALIZE) & Controle (CONTROL) \\
17 & Seleção (SELECT/ & Controle (CONTROL) \\
& DESELECT PRINTER) & \\
$18-25$ & Terra (GROUND) & Terra (GROUND) \\
\hline & &
\end{tabular}

Apesar da porta paralela ser o dispositivo que se comunica com o exterior, ela não foi projetada para controlar dispositivos de potência, sendo necessária uma interface ou "driver" de potência. Esta interface nada mais é que um amplificador de potência para os sinais emitidos pela porta paralela. Como no caso do "dip-coating" temos que controlar apenas um motor de passo, será necessário usar somente os pinos 2-5 da porta paralela (4 pinos de dados), uma vez que cada pino irá controlar o estado de uma das bobinas ("1" ou " 0 ", de acordo com o apresentado na Figura 3 e Tabela 1).

A interface foi construída usando-se o circuito integrado ULN2803, que é composto por oito transistores Darlington em um único chip de 20 pinos. Isto torna a montagem bem compacta. A Figura 4 mostra o esquema eletrônico do controle do motor, onde D0-D3 são conectados aos pinos 2-5 para receber os dados do computador.

Os transistores Q1-Q4, que fazem parte do ULN2803, fornecem a potência elétrica necessária ao motor e protegem o computador de eventuais sobrecargas. 


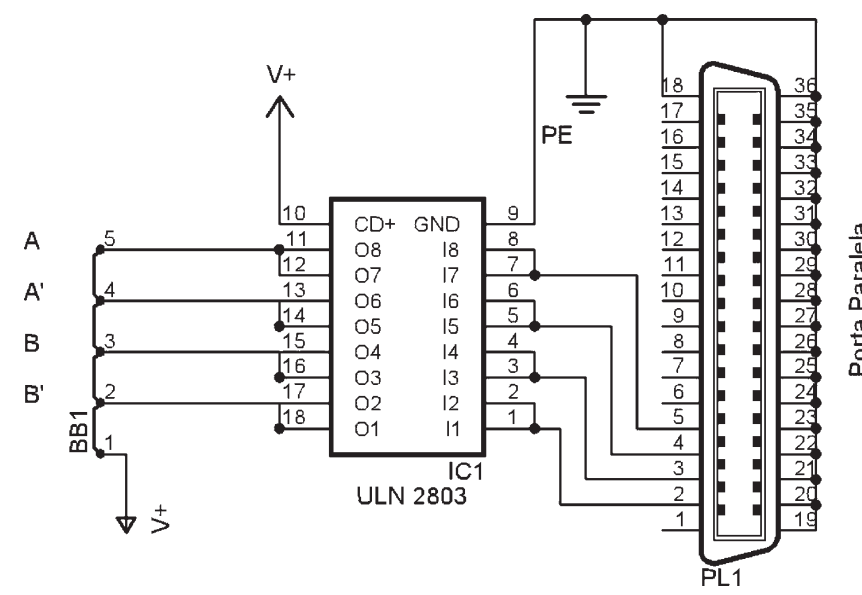

Figura 4. Esquema eletrônico da interface de controle (driver de potência)

\section{O software}

O software que controla este equipamento foi escrito em MSQbasic $^{\circledR}$ e o programa roda no MSDOS $^{\circledR}$, podendo ser instalado e operado em um computador do tipo PC 486. Esta linguagem é fácil e permite o controle da porta paralela de maneira simples.

A porta paralela normalmente é instalada no endereço \&378 em hexadecimal, o que corresponde ao endereço 888 decimal. Assim, ao escrever o comando abaixo no editor do compilador Qbasic ${ }^{\circledR} \mathrm{e}$ executar, o programa faz com que a impressora escreva a letra "A".

OUT 888,65 (65 é o código decimal para a letra "A").

Por outro lado, nosso interesse é enviar dados apenas para os pinos 2-5. Usando a tabela lógica apresentada anteriormente (Tabela 1), teríamos que escrever a sequiência de dados binários: 1000, 0100, 0010, 0001. Para tornar a programação mais simples, convertemos os números binários em números decimais (usando a calculadora científica do Windows ${ }^{\circledR}$ ) e obtemos a seguinte seqüência: 8, 4, 2, 1.

Como são necessários 200 pulsos para completar uma volta, basta repetirmos a seqüência de pulso 50 vezes. Assim, o programa tem o seguinte núcleo de comando:

DIM A(4)

$\mathrm{A}(1)=8 ; \mathrm{A}(2)=4 ; \mathrm{A}(3)=2 ; \mathrm{A}(4)=1$

FOR I=1 TO 50

FOR $\mathrm{P}=1 \mathrm{TO} 4$

OUT $888, \mathrm{~A}(\mathrm{P})$

\section{NEXT P}

NEXT I

OUT 888,0

\section{Seqüência de pulso}

Controle do número de pulso

Index dos "bits" A(1), A(2), .... A $(\mathrm{P})$

Imprime o pulso correspondente $\mathrm{A}(\mathrm{P})$

Avança o index $\mathrm{P}$

Avança o index I

Zera o conteúdo da porta paralela no fim do processo.

Para que o motor gire uma volta e meia basta que seja atribuído o valor 75 à variável I. Desta maneira é possível controlar com precisão a posição da rosca no parafuso.

\section{A montagem}

Para a montagem do equipamento usamos os seguintes materiais:

1) uma base de microscópio antigo que é pesada e confere estabilidade ao equipamento;

2) uma guia linear retirada de uma impressora Deskjet 500;

3) um motor de passo retirado de um "floppy disk" de 5" $1 / 4$;

4) um parafuso com 13 fusos/cm e 4 mm de diâmetro;
5) parafusos, roscas, cola, caixinha de metal para acomodar a eletrônica, etc;

6) uma fonte de alimentação e um cabo de impressora para conectar a interface ao computador.

Para a montagem da interface foi usada a placa de circuito impresso mostrada na Figura 5. O equipamento completo pode ser visualizado na Figura 6.

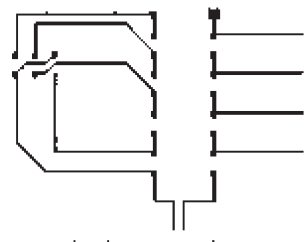

Lado cobreado

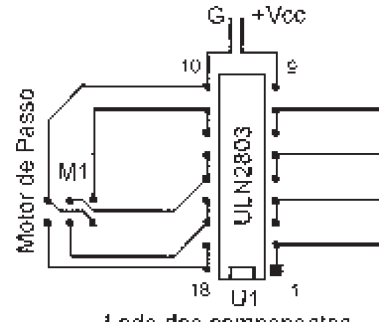

Lado dos componentes
Figura 5. Layout da Interface (placa de circuito impresso)
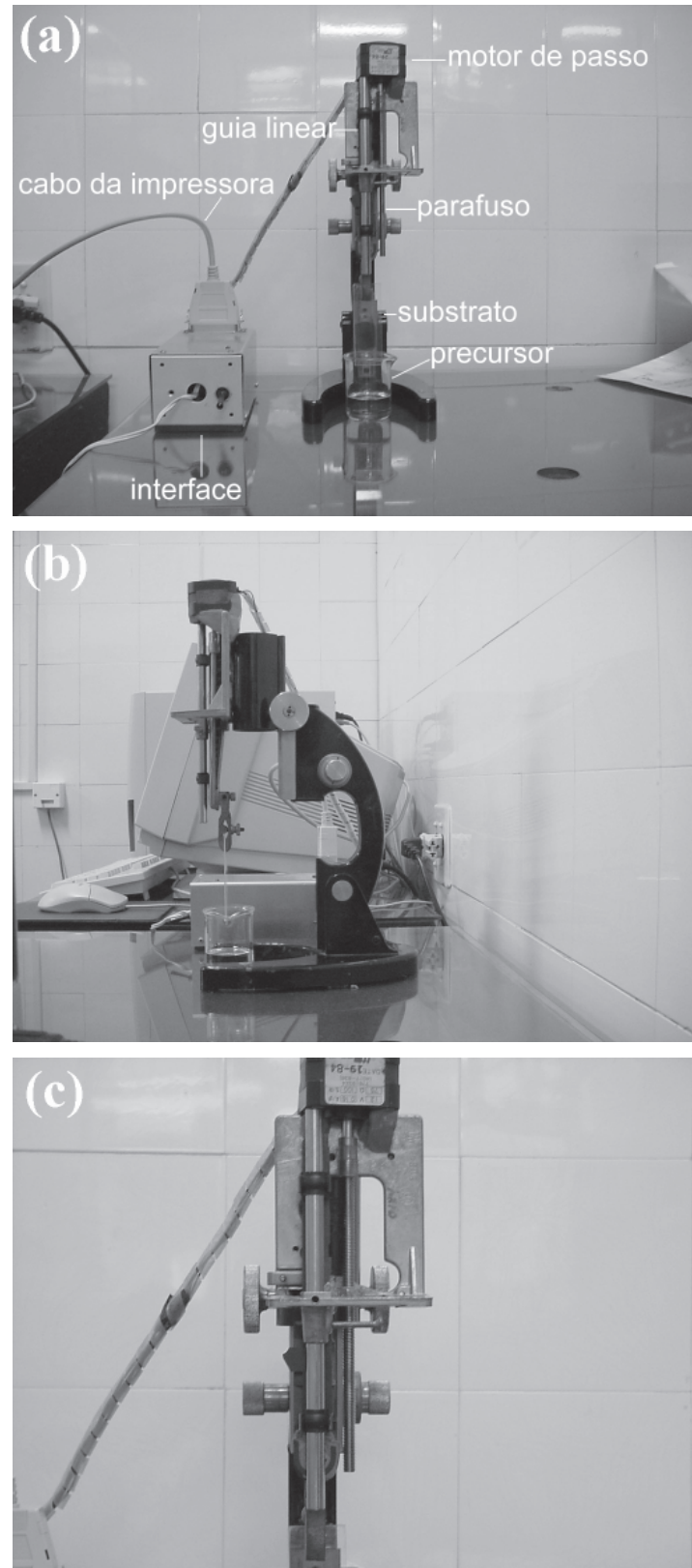

Figura 6. (a) Vista frontal do equipamento; (b) vista lateral do equipamento; (c) detalhe do motor de passo, guia linear e parafuso 


\section{CONCLUSÃO}

Neste trabalho apresentamos um método simples de construção de um equipamento com características muito interessantes para a obtenção de filmes pela técnica de "dip coating", utilizado-se materiais baratos e re-aproveitados a partir de sucatas. Através de um interfaceamento e um programa de controle, é possível otimizar o processo de deposição pelo controle fino da velocidade de descida e subida do substrato e do tempo de imersão na solução precursora. $\mathrm{O}$ equipamento cuja construção foi descrita neste trabalho está em operação desde o início de 2000 e tem sido utilizado na obtenção de filmes de qualidade de diferentes tipos de materiais, como polímeros condutores e nanopartículas metálicas.

\section{AGRADECIMENTOS}

Os autores agradecem ao CNPq e à Rede de Materiais Nanoestruturados (CNPq/MCT) pelo auxílio financeiro.

\section{REFERÊNCIAS}

1. Alves, O. L.; Ronconi, C. M.; Galembeck, A.; Quim. Nova 2002, 25, 69.

2. Hampden-Smith, M. J.; Kodas, T. T.; Ludvikson, A. Em Chemistry of Advanced Materials: An Overview; Interrante, L.; Hampden-Smith, M. J., eds.; Wiley-VCH, Inc, 1998, p. 143.

3. Campbell, D. S.; Thin Solid Films 1976, 32, 3.

4. Vest, R. W.; Ferroeletrics 1990, 102, 53.

5. Nielsen, T. P.; De Guire, M. R.; Solid State Ionics 2000, 151, 61.

6. Galembeck A.; Tese de Doutorado, Universidade Estadual de Campinas, Brasil, 1998.

7. Crowell, J. E.; J. Vac. Sci. Technol., A 2003, 21, S88.

8. Groth T.; Lendlein, A.; Angew. Chem., Int. Ed. 2004, 43, 926. 